(C) [2008] IEEE. Reprinted, with permission, from [Y.Tran, N. Wijesuryia, R.A. Thuraisingham, A. Craig, and H.T. Nguyen, Increase in regularity and decrease in variability seen in electroencephalography (EEG) signals from alert to fatigue during a driving simulated task, Engineering in Medicine and Biology Society, 2008. EMBS 2008. 30th Annual International Conference of the IEEE, 20-25 Aug. 2008]. This material is posted here with permission of the IEEE. Such ermission of the IEEE does not in any way imply IEEE endorsement of any of the University of Technology, Sydney's products or services. Internal or personal use of this material is permitted. However, permission to reprint/republish this material for advertising or promotional purposes or for creating new collective works for resale or redistribution must be obtained from the IEEE by writing to pubs-permissions@ieee.org. By choosing to view this document, you agree to all provisions of the copyright laws protecting it 


\title{
Increase in regularity and decrease in variability seen in electroencephalography (EEG) signals from alert to fatigue during a driving simulated task
}

\author{
Y.Tran, N. Wijesuryia, R.A. Thuraisingham, A. Craig, and H.T. Nguyen ${ }^{\#}$, Senior Member, IEEE
}

\begin{abstract}
Driver fatigue is a prevalent problem and a major risk for road safety accounting for approximately $20-40 \%$ of all motor vehicle accidents. One strategy to prevent fatigue related accidents is through the use of countermeasure devices. Research on countermeasure devices has focused on methods that detect physiological changes from fatigue, with the fast temporal resolution from brain signals, using the electroencephalogram (EEG) held as a promising technique. This paper presents the results of nonlinear analysis using sample entropy and second-order difference plots quantified by central tendency measure (CTM) on alert and fatigue EEG signals from a driving simulated task. Results show that both sample entropy and second-order difference plots significantly increases the regularity and decreases the variability of EEG signals from an alert to a fatigue state.
\end{abstract}

Keywords - driver fatigue, fatigue, electroencephalography (EEG), countermeasure, nonlinear analysis, sample entropy, second-order difference plot, central tendency measure (CTM).

\section{INTRODUCTION}

$\mathrm{F}_{\mathrm{b}}$ atigue is a prevalent problem in society and is known to be related to increased risks of accidents in the workplace and on the road [1]. Driver fatigue is a major risk for road safety as it is associated with decreased concentration, increased reaction time (taking longer to respond) and increased error rate and therefore fatiguerelated accidents can cause severe damage and higher fatalities [2]. Measures have been implemented to reduce fatigue-related road accidents and these include increasing driver awareness through media, setting up driver rest areas and improving road conditions. Despite these efforts, fatigue related accidents still remain one of the main barriers to road safety accounting for up to $40 \%$ of all motor vehicle accidents. One strategy to reduce fatigue-related motor accidents is the use of countermeasure devices. These devices have been categorized into two types (1) those that

Manuscript received April, 6th, 2008. This work was supported in part by ARC grants (LP0560590, DP0666942).

Y Tran is with the Key University Research Centre in Health Technologies at the University of Technology, Sydney, Australia

R. Thuraisingham and N. Wijesuryia are with the Department of Medical and Molecular Bioscience at University of Technology, Sydney, Australia

A.Craig is with the Rehabiliation Studies Unit, Faculty of Medicine at the University of Sydney, Australia.

\#H.T Nguyen is with the Electrical Engineering Department, University of Technology, Sydney, Australia. (phone: 6129514 2451; fax: 6129514 2868; e-mail: Hung.Nguyen@uts.edu.au). measure driving performance and (2) those that measure physiological changes. By providing on-line monitoring of changes in performance or physiological states as well as alerting drivers to these changes, real-time detection of driver fatigue can occur. A problem with countermeasure devices that detect driver performance (i.e. driving errors) is that it is not necessarily a measure of the occurrence of fatigue but a result of the level of experience of the driver [3]. The countermeasures that measure physiological signals are considered more reliable as they provide a direct measure of physiological changes as the driver becomes fatigued. However, one major disadvantage of using physiological changes is the large individual differences in the signals and hence inconsistent results.

Research in using physiological signals as fatigue countermeasures have mainly focused on two types of measures, that is, changes of eye movement and brain activity associated with fatigue. Eye movement countermeasures detect changes such as increased blink rates, eyelid closure rates, droopy eyelids, head nodding etc., through some form of camera/video recording. Although successfully implemented as prototypes in vehicles, one major problem with eye activity monitoring devices is the difficulty in using this method while wearing eyewear/sunglasses, and difficulty in adapting to different light conditions [4]. Another issue with eye activity measures is that changes in eye movements occur at a late stage of fatigue, and therefore cannot solely be relied upon to provide early warnings, which is a crucial feature in any successful driver fatigue alerting/monitoring device [4]. Given the close association of brain activity (using EEG) with human consciousness and the fast temporal resolution, EEG as a measure can be used to provide an early indication of the onset of fatigue. EEG has been shown to measure accurately changes in brain activity in different physiological stages such as drowsiness and fatigue $[5,6]$.

Countermeasures using EEG-based activity have focused on the changes in the spectral activity of different frequency bands during fatigue. For instance, increased theta (3.5$7.5 \mathrm{~Hz})$ and alpha $(8-13 \mathrm{~Hz})$ with reduction in beta $(>13 \mathrm{~Hz})$ is often associated with the onset of sleep [7]. However, the evaluation of spectral activity during fatigue has not led to any validated countermeasure devices as the spectral EEG of an alert individual has been shown to be varied and influenced by many psychological phenomena's [7]. Also, 
algorithms for fatigue countermeasures using the spectral analysis of EEG have often been inconsistent and complex as it involves the interpretation of the interaction of up to four frequency bands [8]. In order to develop a method that can increase the accuracy of successful detection of physiological states like the onset of fatigue during driving, the result of the method implemented needs to be simplified, yet remain precise. In this paper we investigate two different nonlinear techniques, sample entropy and the degree of variability in second-order difference plots quantified by using a central tendency measure (CTM), to investigate differences in the EEG time series between the different physiological states of alertness and fatigue. Both of these nonlinear techniques can yield a single value per time as opposed to four measures accounting for changes in the major frequency bands in spectral EEG. Implementing these methods may presumably have major implications for driver fatigue countermeasure devices.

\section{METHODS}

\section{A. Participants}

Thirty-six participants (19 males, 17 females) were entered into the study. The mean age for male participants were 29.7 (SD 11.4) and for female participants 32.1 (SD 12.5).

\section{B. Experimental Tasks}

Participants performed a driving simulator task and were asked to drive along a straight road with minimal stimulation aimed at reducing their cognitive load and leading them towards boredom and fatigue. Simulated driving tasks are used in fatigue studies, both in clinical and research settings as they provide a measure to detect fatigue by means of decreased vigilance [9]. In this study we used the Divided Attention Steering Simulator (DASS) (Stowood Scientific Instruments), according to the manufacturer's instructions. The DASS has been used reliably in other studies [9]. The program measures decreasing vigilance/ fatigue, by the means of increased deviation in the steering the car and changes in driver reaction time. The aim of the task was to steer the car down the centre of the road on the computer screen using a gaming steering wheel (Grandprix 1, Thrustmaster, USA). During the task, digits that changed randomly were displayed at the corner of the screen. To test vigilance and reaction time the subjects were required to identify the number "2" when it appeared by pressing a button on the same side of the steering wheel as it appeared on the screen. The task was considered monotonous because the participants were required to drive on a straight road at a constant slow speed for an extended period of time in a noise, stimulus and temperature controlled laboratory.

\section{Data Acquisition and Pre-processing}

The Biosemi ${ }^{\mathrm{TM}}$ Active-Two System was used in this study for recording EEG signals from each subject. Raw data was acquired using 32-channels at a sampling rate of $2048 \mathrm{~Hz}$ with 24-bit digital resolution per channel, using the biopotential measurement system and active electrodes. The 32-channels were recorded following the International 10-20 Montage system and the channels numbers (1-32) are in the following order (FP1, AP3, F7, F3, FC1, FC5, T7, C3, CP1, CP5, P7, P3, PZ, PO3, O1, OZ, O2, PO4, P4, P8, CP6, CP2, C4, T8, FC6, FC2, F4, F8, AF4, FP2, FZ, CZ). The signal was down sampled to $256 \mathrm{~Hz}$. The recording of the EEG signals was conducted in a dedicated temperature controlled research laboratory. Several pre-processing steps were also performed on the raw EEG prior to nonlinear analysis using sample entropy and CTM of the second order difference plots. A high pass filter was applied at $1 \mathrm{~Hz}$ to remove movement artifact (drift in the electrical signals). In addition any artifact such as eye and muscle movements, which are non-cerebral in origin, were removed from the recorded EEG data using Independent Component Analysis (ICA) using EEGLab [10].

Two sets of 1-minute EEG data were taken and analyzed from an alert state during the start of the task where the participants were engaged to the task but not fatigued and during fatigue as determined by video monitoring, physiological symptoms and performance decrements from the driving task.

\section{Analysis-Sample Entropy and Second-order Difference Plots}

Sample Entropy: We used sample entropy to measure complexity. Below is a brief description of the evaluation of sample entropy.

Consider the time series $x(i), i=1,2,3, \ldots, N$. Let us choose the two input parameters $m$ and $r$, where $m$ is the vector length and $r$ the criterion of similarity. Let the $m$ samples beginning at sample $x(i)$ be denoted by the vector $v_{m}(i)$ $[=x(i), x(i+1), \ldots, x(i+m-1)]$ and consider the set of all vectors of length $m$ within $x(n)$, that is $\left[v_{m}(1), v_{m}(2) \ldots \ldots . . v_{m}(N-m)\right]$. Let us define [11]

$$
B_{i, m}(r)=\frac{n_{i, m}^{\prime}(r)}{N-m-1}
$$

where $n_{i, m}^{\prime}(r)$ is the number of vectors that are similar to $v_{m}(i)$, given the similarity criterion $r$, excluding self matching. Similar calculations are carried out for each $i$, with $i=1,2 \ldots N-m$. The function $B_{m}(r)$ is then defined which is the average of the function $B_{i, m}(r)$ [11],

$$
B_{m}(r)=\frac{\sum_{i=1}^{N-m} B_{i, m}(r)}{N-m}
$$

Similarly let $B_{i,(m+1)}(r)$ be defined as

$$
B_{i,(m+1)}(r)=\frac{n_{i,(m+1)}^{\prime}(r)}{N-m-1}
$$


where $n_{i,(m+1)}^{\prime}(r)$ is the number of vectors in the sequence $\left[v_{m+1}(1), v_{m+1}(2) \ldots \ldots . . v_{m+1}(N-m)\right]$ that are similar to $v_{(m+1)}(i)[=x(i), \quad x(i+1), \ldots, x(i+m)], \quad$ given the similarity criterion $r$, excluding self matching. Similar calculations are carried out for each $i$, with $i=1,2 \ldots N-m$. The function $B_{(m+1)}(r)$ is then defined which is the average of the function $B_{i,(m+1)}(r)$.

The statistic $\operatorname{SampEn}(m, r, N)$ is then defined by [11],

$$
\operatorname{SampEn}(m, r, N)=-\ln \left(B_{m+1}(r) / B_{m}(r)\right)
$$

This is the negative natural logarithm of the conditional probability that two sequences similar for $m$ points remain similar at the next point, where self matches are not included in calculating the probability. A lower value of SampEn indicates more self similarity in the time series. The system parameter $\operatorname{SampEn}(m, r)$ is then defined by [11],

$$
\operatorname{SampEn}(m, r)=L t_{N \rightarrow \infty} \operatorname{SampEn}(m, r, N)
$$

Based on the work of Pincus [12] values of $m=2$ and $r$ equal to $15 \%$ of the standard deviation (std. dev) of the data sets were chosen in this work. The value of $N$ chosen, is 7680 which is more than sufficient to estimate $\operatorname{SampEn}(m, r)$

Second-order Difference Plots quantified by Central Tendency Measure (CTM): In the second order difference plot, $(x(\mathrm{n}+2)-\mathrm{x}(\mathrm{n}+1))$ is plotted against $(x(\mathrm{n}+1)-x(\mathrm{n}))$. It is a plot of successive rates against each other and gives a graphical representation of the rate of variability: $(x(\mathrm{n}+2)-$ $x(\mathrm{n}+1))$ versus $(x(\mathrm{n}+1)-x(\mathrm{n}))$. It shows the rate of variation of successive rates. For continuous data as the sampling interval decreases, the rates scale appropriately and approach the result of the continuous data. Therefore, provided the sampling interval is small, the differences will approach a scaled rate of the continuous function where the scaling factor is the sampling frequency [13].

The central tendency measure quantifies the variability seen in the second order difference plot. It is computed by selecting a circular region of radius $r$, around the origin, counting the number of points that fall within the radius, and dividing by the total number of points. Let $n$ be the total number of points and $r$ the radius of the central area. Then,

$$
C T M=\left[\sum_{i=1}^{n-2} \delta\left(d_{i}\right)\right] /(n-2)
$$

$$
\delta\left(d_{i}\right)=1 \quad \text { if }\left([x(i+2)-x(i+1)]^{2}+[x(i+1)-x(i)]^{2}\right)^{0.5}<r
$$

$=0 \quad$ otherwise

For each radius $r$, CTM provides the fraction of the total number of points that lie within it. Sample values, which are within a small radius $r$, correspond to successive rates that are small. These reflect the low frequency components or the slow ascending, descending part of the function in the time series. Sample values that correspond to high $r$ are those of high frequency components or the rapid ascending, descending part of the function in the time series. For a particular radius $r$, CTM counts the number of successive rates that have all sign combinations, without any distinction. In order that we do not count sample values corresponding to spurious high frequency noisy components, the radius $r$, when the CTM reaches a value 0.90 is chosen as a measure of the total variability of the system. This measure of central tendency was found to be better than the measurement based on the average length, which tends to neglect the fine structure distribution [13].

$$
\frac{1}{(n-2)} \sum_{i=1}^{(n-2)}\left([x(i+2)-x(i+1)]^{2}+[x(i+1)-x(i)]^{2}\right)^{0.5}
$$

\section{RESULTS}

\section{A. Sample Entropy}

The sample entropy values were reduced from the alert to the fatigue condition in all 32-channels for the 36 participants (Fig 1). Using dependent t-test analyses, this was found to be significantly different $(p<0.05)$ for the EEG sites FP2, F8, FZ, FC1, FC5, FC6, C3, CZ, CP1, CP5, CP6, T7, T8, P3, P7, P8, PO3, O1, O2 and OZ. This shows a global decrease in complexity in all the cortical regions on the scalp.

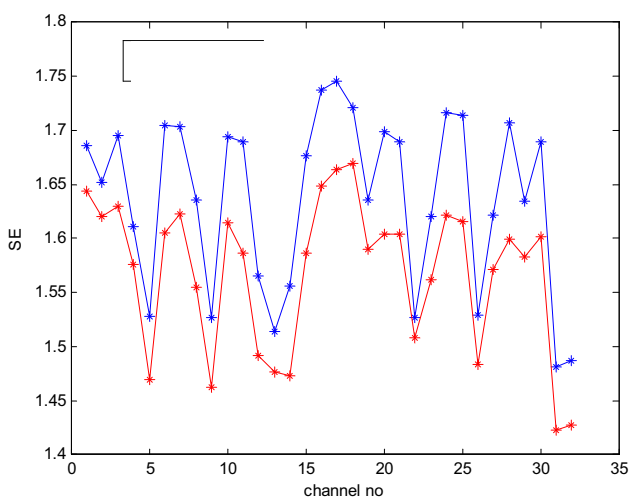

Fig 1: Shows the mean sample entropy values from 36 participants during alert (Top line) and fatigue (bottom line) conditions. Sample entropy was reduced in all 32 channels.

\section{B. Second-order Difference Plots}

Fig. 2 shows CTM values as a function of $\mathrm{r}$ (radius) for alert (blue line) and fatigue (red line) conditions. For CTM of 0.9 , the radius in which most of the values are situated, there is a reduction in variability seen in the fatigue condition compared with the alert condition.

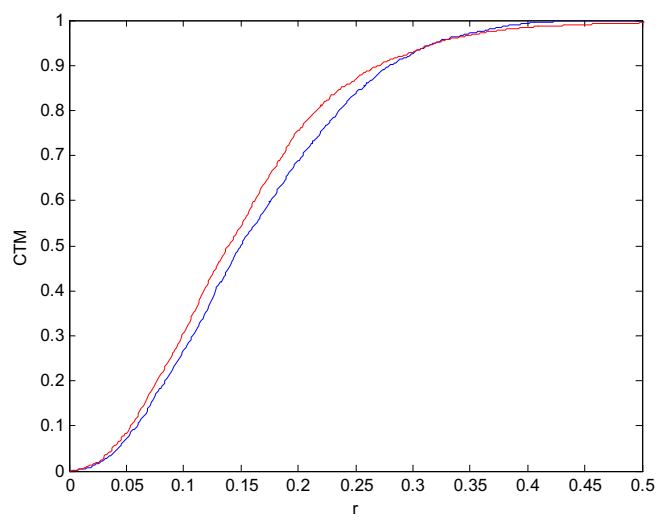


Fig 2: Shows the CTM values as a function of $r$ (radius) from alert (blue line) to fatigue (red line) in all 36 participants.

The CTM values were reduced from the alert to fatigue condition in 31 of the 32 channels in the 36 participants (Fig $2)$. Using related t-test analyses, CTM values were found to be significantly different $(\mathrm{p}<0.05)$ for the EEG sites FP2, F7, F8, FC5, C3, CP5, CP6, T7, T8, P3, P7, and P8. This finding suggests a decrease in the variability in the signal in frontal, central and parietal cortical regions on the scalp.

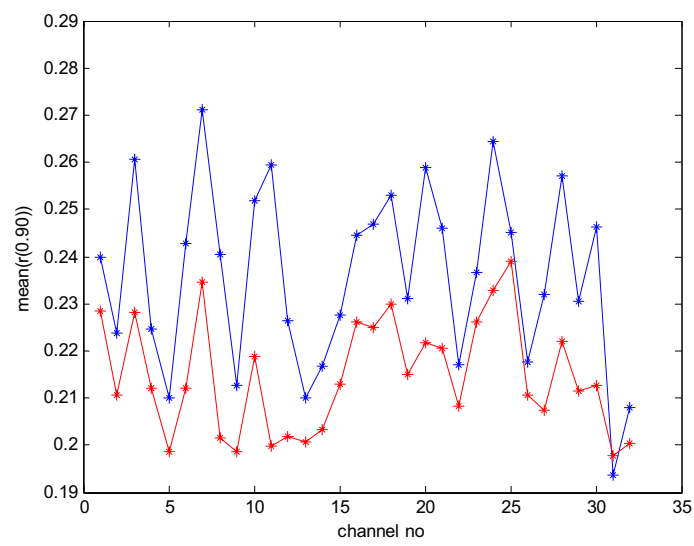

Fig 3: Shows the mean CTM values from 36 participants during alert (Top line) and fatigue (bottom line) conditions. CTM was reduced in 31 channels.

\section{DISCUSSION}

The results show significant reduction in sample entropy in all regions of the EEG 32-channels from the alert to fatigue condition, following a driver simulated task. This finding suggests that there is an increase in regularity in the EEG signals associated with the person becoming fatigued. Reduction of irregularity has been found in other studies examining sample entropy changes during a mental task state before and after physical exertion [14]. These investigators also found a sharp decrease in sample entropy values after the physical exertion where the participants were believed to be fatigued. This is also consistent with sleep studies that analyze sleep EEG by sample entropy and which has found marked reduction in irregularity as the participants move into deeper stages of sleep [15].

Second-order difference plots quantified by CTM also significantly reduced from the alert to fatigue state. This technique measures the degree of variability seen in a second-order difference plot. A second-order plot is similar to a Poincare plot where instead of plotting successive values of the time series against each other, successive differences in the time series are plotted against each other [13]. These differences approximate to the first derivatives in a uniformly sampled time series. Therefore the CTM measures the variability in this plot. This degree of variability has been shown to be useful in the study of chaotic systems [16] and in this study, signal variability was significantly reduced associated with fatigue.

\section{CONCLUSION}

EEG irregularity and variability was found to have reduced significantly from an alert state to a fatigued state. These results are exciting as it reveals that fatigue can be quantified using nonlinear analysis. Unlike the spectral analysis of EEG signals, typically used in analyzing EEG signals, the results are easier to interpret as it yields one value as opposed to interpreting the interactions of several frequency bands. These nonlinear measures can potentially be implemented in countermeasures that detect changes in brain activity during changes in physiological states to prevent problems like driver fatigue, and could result in the application of this method to improve road safety.

\section{REFERENCES}

[1] J. T. Arnedt, M. A. C. Geddes, A. W. Maclean "Comparative sensitivity of a simulated driving task to self-report, physiological, and other performance measures during prolonged wakefulness", J. Psychosom. Res., vol. 58, pp.61-71, 2005.

[2] Australian Transport Safety Bureau, "Road safety research report OR 23. Fatigue related crashes: An analysis of fatigue-related crashes on Australian roads using an operational definition of fatigue" [online] $<$ http://www.atsb.gov.au/publications/2002/pdf/Fatigue related sum.pdf $>$

[3] A. W. MacLean, D. R. T. Davies, and K. Thiele, "The hazards and prevention of driving while sleepy" Sleep. Med. Rev. vol. 7, pp 507-521, 2003.

[4] A. Williamson, and T. Chamberlain. "Review of on-road driver fatigue monitoring devices" NSW Injury Risk Management Research Centre, University of New South Wales, Australia., April Available at: http://www.maa.nsw.gov.au/getfile.aspx? Type $=$ document $\& I D=3988 \&$ Obje ct Type $=3 \&$ ObjectID $=715,2005$.

[5] G. Kecklund and T. Akerstedt, "Sleepiness in long distance truck driving: An ambulatory EEG study of night driving" Ergonomics, vol.36, pp. 1007-1017, 1993

[6] S. Makeig and T. Jung. "Changes in alertness are a principle component of variance in the EEG spectrum", NeuroReport, vol.7 pp. 213-216, 1995.

[7] R. Bittner, K. Hana, L. Pousek, P. Smrha, P. Schreib and P. Vysuky. "Detecting of fatigue state of a car driver", Lect. notes Comp. Sci., vol.1933, pp123-126, 2000.

[8] S. Lal, A. Craig, P. Boord, L. Kirkup, and H. Nguyen "Development of an algorithm for an EEG-based driver fatigue countermeasure", J. Safety Res., vol. 34, pp. 321-328, 2003.

[9] P. Philip, J. Taillard, E. Klein, P. Sagaspe, A. Charles, W. L. Davies, C. Guilleminault, B. Bioulac "Effect of fatigue on performance measured by a driving simulator in automobile drivers", J. Psychosom. Res., vol. 55, pp.197-200, 2003

[10] A. Delorme and S. Makeig "EEGLab: an open source toolbox for analysis of single-trial EEG dynamics including independent component analysis", J Neurosci. Methods, vol.134, pp. 9-21, 2004.

[11] J. S. Richman and J. R. Moorman, "Physiological time-series analysis using approximate entropy and sample entropy", Am. J. Physiol. (Heart Circ.Physiol.), vol. 274, pp. H2039-H2049, 2000.

[12] S. M. Pincus "Approximate entropy as a measure of system complexity”, Proc. Natl. Acad. Sci., USA, vol., 88 pp. 2297-2301, 1991.

[13] R. A. Thuraisingham, Y. Tran, P. Boord and A. Craig "Analysis of eyes open, eye closed EEG signals using second-order difference plot", Med.Biol. Eng. \& Comp., vol.45, pp.1243-1249, 2007.

[14] L. J.Trejo, R. Kochavi, K. Kubitz, L. D. Montgomery, R. Rosipal, and B. Matthews "EEG-based estimation of cognitive fatigue", Proc. SPIE, vol.5797, pp.105-115, 2005.

[15] W. X. He, X. G. Yan, X. P. Chen, and H. Liu "Nonlinear feature extraction of sleeping EEG signals", Proc. 2005 IEEE Eng. Med. Biol. pp 4614-4617, 2005.

[16] M. E. Cohen, D. L. Hudson, and P. Deedwania "Applying continuous chaotic modeling to cardiac signal analysis" IEEE Eng. Med. Biol. Mag. Vol.15, pp. 97-102, 1996. 